\title{
Denis Diderot, Lettres à Sophie Volland. 1759-1774
}

\section{Paolo Quintili}

\section{(2) OpenEdition}

\section{Journals}

\section{Edizione digitale}

URL: https://journals.openedition.org/studifrancesi/4698

DOI: $10.4000 /$ studifrancesi.4698

ISSN: 2421-5856

\section{Editore}

Rosenberg \& Sellier

\section{Edizione cartacea}

Data di pubblicazione: 1 avril 2012

Paginazione: 151-152

ISSN: 0039-2944

\section{Notizia bibliografica digitale}

Paolo Quintili, «Denis Diderot, Lettres à Sophie Volland. 1759-1774», Studi Francesi [Online], 166 (I | LVI) |

2012, online dal 30 novembre 2015, consultato il 19 novembre 2021. URL: http://

journals.openedition.org/studifrancesi/4698 ; DOI: https://doi.org/10.4000/studifrancesi.4698

Questo documento è stato generato automaticamente il 19 novembre 2021.

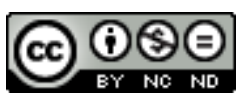

Studi Francesi è distribuita con Licenza Creative Commons Attribuzione - Non commerciale - Non opere derivate 4.0 Internazionale. 


\title{
Denis Diderot, Lettres à Sophie Volland. 1759-1774
}

\author{
Paolo Quintili
}

\section{NOTIZIA}

DENIS DIDEROT, Lettres à Sophie Volland. 1759-1774, édition présentée et annotée par Marc BUfFAT et Odile RICHARD-PAUCHET, Paris, Non Lieu, 2010, pp. 715.

Questa nuova edizione della corrispondenza amorosa tra Denis Diderot (1713-1784), le Philosophe, e la sua amante Louise-Henriette Volland (1716-1784), ribattezzata Sophie «alla culla della filosofia», viene a colmare un vuoto editoriale che durava da oltre un quarantennio. Dopo la prima edizione, parziale e lacunosa, a cura di André Babelon, per Gallimard negli anni '30 del secolo scorso (riedita nel 1978) e l'ormai introvabile edizione Les lettres de Diderot à Sophie Volland, presso Le Club français du Livre, a cura di Yves Florenne (Paris, 1965), il lettore e lo studioso non disponevano più di una raccolta integrale di questo epistolario che è considerato, a giusto titolo, una delle vette più alte della letteratura occidentale nel genere. Jean Varloot ne ha fornito un'edizione parziale ma ben mirata dal punto di vista filosofico (Lettres à Sophie Volland, Paris, Gallimard, 1984) e che tuttavia non rende la complessità della liaison. Restano, certo, la monumentale edizione della Correspondance completa di Diderot, a cura di G. Roth (16 voll., Paris, Minuit, 1955-1970), o i quindici volumi delle Euvres complètes a cura di R. Lewinter (1969-1973) o i cinque dell'edizione L. Versini (1994-1997), in cui il lettore può ritrovare, sparse, le 187 lettere - alcune dei veri e propri racconti o apologhi filosofici lunghi una decina di pagine - del solo Philosophe. Le lettere di Louise-Henriette-Sophie sono andate perdute. L'interminabile edizione «ufficiale» delle Euvres complètes de Diderot, a cura di H. Dieckmann, J. Proust e J. Varloot (33 voll., Paris, Hermann, 1975-), attenderà ancora a lungo, prima di offrire al pubblico gli ultimi tre volumi della Correspondance. 
2 Il pregio, dunque, della nuova edizione è di costituire un grande evento letterario nel pieno senso del termine. I curatori hanno ripreso i due tomi dei manoscritti autografi diderotiani, parte del Fonds Vandeul, O. Richard-Pauchet ha stabilito il testo e ciascuna lettera è corredata ora di un ricco apparato critico di commento e di varianti (correzioni, letture ipotetiche, passaggi soppressi ecc.). Marc Buffat fa precedere le lettere da un ricco saggio introduttivo (Une Correspondance amoureuse), in cui espone le peculiarità filosofiche di questa scrittura epistolare: «La lettre comme don de soi», «Pour autrui», «La musique dans les lettres», ecco le tappe della lettura/scrittura diderotiana in atto (pp.7-24). Odile Richard-Pauchet perfeziona il commentario con una Postfazione dal titolo: Lettres à Sophie Volland. L'œeuvre inadvertante (pp. 665-676), insistendo sulla reciprocità del lavoro filosofico: l'amante non perverrebbe a una tale chiarezza di concetti e d'intenti filosofici senza l'«avvertenza», la vigile presenza dell'altra. Il pensiero si plasma attraversando «Le moule de la pensée» (primo paragrafo della Postfazione, pp.665-671), il modello, lo «stampo» di un pensiero comune, considerato anche «Du côté de chez Sophie: un style» (pp.672-674), dalla parte di lei si sviluppa precisamente «Du romanesque dans les lettres» (pp.674-676), il piglio letterario tipico della scrittura diderottiana décousue, frammentata e sciolta, quella del romanzo e del conte. I saggi dei curatori sono infine accompagnati da una ricca Appendice con annesse informazioni su Sophie, la famiglia Diderot, la famiglia Volland, l'ambiente enciclopedico $\mathrm{e}$, più in generale, sulla cerchia intellettuale e personale di Diderot, seguite da un utile apparato iconografico: il Plan Turgot (1739) di Parigi, il quartiere del Palays-Royal e il «carré magique» di Sophie Volland e di Diderot (pp. 688-689).

3 Come definire l'impresa della corrispondenza Denis-Sophie, nel suo insieme ? Qualcuno ha voluto parlare, pour cause, di «dialogo a una voce» (J. CHouilLET, Denis Diderot-Sophie Volland. Un dialogue à une voix, Paris, Honoré Champion, 1986), poiché la situazione del testo lascia indovinare al lettore cosa ci sarebbe dall'altra parte del foglio, dalla parte di Sophie, pure assente; ma indicando ambiguamente la straordinaria sintonia di affetti che ha caratterizzato l'amicizia e l'amore di una vita. Lo scambio epistolare si ferma al 3 settembre 1774, tappa dell'avventuroso viaggio di ritorno di Diderot dalla Russia di Caterina II, passando per l'Olanda, all'Aia, di cui ci resterà un vivido ritratto nel Voyage en Hollande. I due amanti vivranno altri dieci anni, si scriveranno ancora, ma Denis non conserverà memoria del dopo. Sophie muore nel febbraio del 1784, l'amico/amante, inseparabile, la seguirà nell'ultimo viaggio il 31 luglio 1784. I due sognavano insieme un destino d'immortalità ipotetica, «materialista», come scrisse poeticamente il filosofo del Rêve de d'Alembert (1769) in una lunga missiva dal castello del barone d'Holbach, al Grandval, il 15 ottobre 1759. È una pagina giustamente celebre, che gli editori commentano con cura: «Le sentiment et la vie sont éternels. Ce qui vit a toujours vécu et vivra sans fin. La seule différence que je connaisse entre la mort et la vie, c'est qu'à présent vous vivez en masse, et que dissous, épars en molécules, dans vingt ans d'ici vous vivrez en détail [...]. Le reste de la soirée s'est passé à me plaisanter sur mon paradoxe. On m'offrait de belles poires qui vivaient, des raisins qui pensaient. Et moi, je disais, Ceux qui se sont aimés pendant leur vie et qui se font inhumer l'un à côté de l'autre ne sont peut-être pas si fous qu'on pense. Peut-être leurs cendres se pressent, se mêlent et s'unissent. Que sais-je? Peut-être n'ont-elles pas perdu tout sentiment, toute mémoire de leur premier état. Peut-être ont elles un reste de chaleur et de vie dont elles jouissent à leur manière au fond de l'urne froide qui les renferme. Nous jugeons de la vie des éléments par la vie des masses grossières! Peut-être sont-ce des choses bien 
diverses. On croit qu'il n'y a qu'un polype; et pourquoi la nature entière ne serait-elle pas du même ordre? Lorsque le polype est divisé en cent mille parties, l'animal primitif et générateur n'est plus, mais tous ses principes sont vivants. Ô ma Sophie, il me resterait donc un espoir de vous toucher, de vous sentir, de vous aimer, de vous chercher, de m'unir, de me confondre avec vous, quand nous ne serons plus! S'il y avait dans nos principes une loi d'affinité, s'il nous était réservé de composer un être commun; si je devais dans la suite des siècles refaire un tout avec vous; si les molécules de votre amant dissous venaient à s'agiter, à se mouvoir et à rechercher les vôtres éparses dans la nature! Laissez-moi cette chimère. Elle m'est douce. Elle m'assurerait l'éternité en vous et avec vous...» (pp. 78-79).

4 Si tratta qui - oltre lo sfogo retorico di un amante che «s'arrabbia di essere impigliato in una diavolo di filosofia [atea e materialista] che la mente non può impedirsi di approvare e il cuore di smentire!» (fine settembre 1769, a un'altra amante: Madame de Maux) -, di una breve esposizione della teoria vitalistica della materia che Diderot sviluppava, in quegli stessi anni, negli Éléments de physiologie (éd. par P. Quintili, Paris, Honoré Champion. 2004) e negli scritti filosofici dell'ultimo periodo (Rêve de D'Alembert, Principes philosophiques sur la matière et le mouvement ecc.). Se ne troveranno spesso di pagine simili, tra le righe delle lettere. In altri casi, è invece questione di rapidi sfoghi, effusioni nell'ombra alla ricerca dello sguardo dell'altra, della sua lumière, dalla profondità del buio di una stanza: «J'écris sans voir. Je suis venu. Je voulais vous baiser la main et m'en retourner (...). Adieu, ma Sophie, bonsoir. Votre cœur ne vous dit-il donc pas que je suis ici. Voilà la première fois que j'écris dans les ténèbres (...). Partout où il n'y aura rien, lisez que je vous aime (10 giugno 1759)» (p. 34). Il gioco dei riflessi e dei reciproci sguardi è una cifra ricorrente della retorica epistolare diderotiana. «J'ai voulu vivre sous vos yeux»: l'esergo, assai ben scelto, dell'Introduzione di Buffat segna il carattere della liaison come di un tentativo di realizzare, a due, una vita «di verità», sotto uno sguardo di reciprocità scelta, condivisa, con tutte le aporie e gli ostacoli che un simile proposito «enciclopedico» dovette incontrare. Osserva Buffat: «L'amoureux passionné se double donc d'un moraliste et d'un savant (...). Une telle démarche implique un sujet séparé de lui-même, s'étudiant comme un objet. Au moyen de l'introspection et au nom d'un humanisme, c'est-à-dire de l'idée d'une unité de la nature humaine, est visée l'élaboration d'une science du sujet amoureux et plus généralement d'une psychologie scientifique, qui serait à la fois introspective et objective, les deux n'étant pas pour Diderot contradictoires. Projet dont l'ampleur ne le cède en rien à celle de l'Encyclopédie» (p. 11). $\mathrm{E}$ in effetti, la varietà dei racconti, la loro bellezza, la profondità di certe interlocuzioni e interrogativi del Philosophe amante, lascia infallibilmente pensare, di fronte all'epistolario Diderot-Volland, a una vera enciclopedia del discorso amoroso, del secolo xviII e oltre.

Nella nostra lingua queste lettere hanno conosciuto il destino che fu del Diderot dei secoli XIX e Xx, quello dell'eterno autore dei morceaux choisis. L'editore Archinto ha fornito un'edizione di testi scelti, a cura di Marina Premoli, pubblicata nel 1990 con il titolo un po' fuorviante ma commercialmente accattivante : Siamo tutti libertini. Lettere a Sophie Volland, 1759-1762 (183 pp.), «arricchita» nel 2010 da una nuova Introduzione di Eugenio Scalfari. Siamo vicini alla soglia del tricentenario diderotiano (2013) ed è auspicabile che sulla base di questa splendida edizione Buffat-Richard-Pauchet venga presto offerta anche al lettore italiano (e agli studi universitari francesi) una nuova immagine del Philosophe, che osi spingersi al di là della cifra un po' consunta del 
«libertino» (in parte giusta, ma impoverente) e che dia pieno conto della dimensione filosofica e letteraria alta delle opere e delle «scritture» dell'autore del Neveu de Rameau. 